\title{
Le concept de modèle scientifique : Vision des étudiants universitaires algériens
}

\section{The concept of scientific model: Vision of Algerian university students}

\author{
Djamel Houatis ${ }^{1 *}$ \\ ${ }^{1}$ Laboratoire de Physique des Techniques Expérimentales et ses Applications, Faculté des Sciences, \\ Université Yahia Fares de Médéa, Algérie
}

\begin{abstract}
Résumé. La notion du "modèle" est devenue un outil indispensable dans le processus d'acquisition des connaissances et l'usage du terme "modèle" est devenu très fréquent dans les discours et les écrits en classe. Dans cet article, nous allons porter notre attention sur l'interaction des étudiants universitaires algériens avec le concept "modèle", nous identifierons les trais distinctifs de leurs points de vue épistémologiques sur la notion de "modèle" en sollicitant par questionnaire des étudiants de trois établissements universitaires algériens (deux universités et une école supérieure). Nous avons choisi de poser des questions fermées avec justification des réponses. La grille d'analyse de nos résultats est basée sur des thèmes centraux préalablement sélectionnés. Sur le plan théorique, nous nous sommes placés dans le cadre du schéma de Jean-Louis Martinand qui décrit l'activité de la modélisation dans la classe. Les résultats de cette étude pourraient, il nous semble, être pris en compte dans la conception des programmes afin de mettre les étudiants dans des conditions favorables lors de l'intégration des modèles dans l'enseignement de la physique.
\end{abstract}

\begin{abstract}
The notion of "model" has become an indispensable tool in the process of acquiring knowledge, and the use of the term "model" has become very common in speeches and writings in class. In this article, we will focus on the interaction of Algerian university students with the concept model, we will identify the distinctive features of their epistemological views on the notion of model, soliciting them by questioning students from three universities, (Yahia Fares University of Medea, Saâd Dahlab University of Blida, Teacher's higher college of Kouba). yes or no questions have been chosen to be asked provided with justifications of answers. The analysis grid for our results is based on previously selected central themes. On the theoretical plan, we have placed ourselves within the framework of JeanLouis Martinand's schema which describes the activity of modelling in the classroom. it seems to us, that the results of this study might be taken into
\end{abstract}

* Corresponding author: houatis.djamel@univ-medea.dz 
consideration when designing the programs in order to put the students in favourable conditions when integrating the models in teaching physics.

\section{Introduction}

La grande majorité des étudiants universitaires prennent actuellement en aversion la pratique de l'enseignement des sciences exactes en particulier la physique [1-3]. En effet, plusieurs étudiants perçoivent la physique comme étant trop difficile et jugent qu'elle est plutôt destinée aux élèves doués ou forts. Différents chercheurs dans le domaine de l'éducation et de la didactique s'interrogent sur les facteurs qui sont à l'origine de cette baisse d'intérêt. L'un des facteurs incriminés est la manière dont ces sciences sont enseignées [4]. La seconde thématique concerne la place des modèles et de la modélisation dans l'enseignement car l'apprentissage des sciences notamment la physique s'appuie nécessairement sur une approche fréquemment basée sur un modèle scientifique [5].

La notion de modèle bien que fort répandue dans tous les champs disciplinaires, possède en sciences physiques une spécificité toute particulière, et sa signification présente une résonnance et une portée propre à cette discipline. De nombreux chercheurs ont contribué de manière significative à la réflexion sur les modèles et la modélisation dans les disciplines scientifiques plus particulièrement en physique [6-10]. Il est notoire qu'il existe un consensus entre tous ces chercheurs scientifiques sur la place centrale occupée par les modèles en sciences [11-19]. L'analyse de l'élaboration des modèles, de leurs statuts et de leurs usages, notamment dans les sciences physiques constitue un terrain d'investigation encore peu exploré, ce qui se reflétait négativement dans l'utilisation des modèles et des démarches de modélisation dans l'enseignement de la physique.

Les modèles sont couramment utilisés dans les cours de physique pour aider à expliquer les concepts scientifiques. Ils ne sont pas nécessairement des objets d'enseignement mais sont souvent implicites dans les discours et les écrits en classe. Plus que jamais, la question du modèle et de la modélisation est d'actualité, l'on parle en effet de la modélisation d'une force par un vecteur, d'un mobile par un point matériel, d'une lumière par un rayon lumineux, d'un modèle atomique, d'un modèle standard... La modélisation dans l'enseignement est l'utilisation des modèles dans la pratique scolaire selon une approche de construction qui implique des choix d'éléments en lien avec le problème traité. La modélisation permet la mise en relation de ces éléments. Elle est "l'essence même d'une pensée et d'un travail scientifique" [20] dont les modèles sont les outils pour penser et mener le projet d'enseignement. L'importance que détiennent les modèles et les démarches de modélisation dans l'enseignement de la physique nous permet d'affirmer que la réussite d'un apprentissage est fonction de l'action efficace de l'enseignant et de sa méthode à diriger les activités des apprenants vers l'utilisation des modèles et de la modélisation en classe. Ainsi pour les étudiants, le modèle est avant tout un outil indispensable dans le processus d'acquisition des connaissances flexibles, transférables et applicables [21-22].

Des recherches antérieures ont montré que l'appréciation des modèles par les étudiants est très limitée et naïve [23-25].

$\mathrm{Au}$ cours des deux dernières décennies, de nombreux travaux ont été réalisés afin de déterminer les points de vue épistémologiques des élèves de l'enseignement primaire et secondaire sur la notion de "modèle". Dans cette étude, nous allons porter notre attention sur l'interaction des étudiants universitaires algériens avec le concept "modèle" dans leurs études en physique, nous examinerons leurs points de vue sur les modèles en physique selon différents indicateurs. Il s'avère que de telles recherches sont encore rares pour l'enseignement supérieur. Les résultats de cette étude serviront à identifier les critères utilisés par les étudiants pour classifier les modèles, et pour cerner les éléments permettant de 
mesurer leurs positions philosophiques. Ils pourraient également nous semble-t-il, être pris en compte dans la conception des programmes afin de mettre les étudiants dans des conditions favorables lors de l'intégration du modèle dans l'enseignement de la physique.

\section{Utiliser la modélisation dans l'enseignement de la physique}

Pour notre analyse en termes de modèle et d'activité de modélisation en physique, nous nous sommes appuyés sur le schéma de Jean-Louis Martinand (Fig. 1.). En partant du constat que les étudiants en situation d'apprendre de la physique doivent connaitre que la physique, en tant qu'archétype des sciences empiriques, se caractérise par son recourt systématique à l'élaboration des modèles, et que l'utilisation du modèle en physique est inévitable, puisqu'il permet de faire le lien entre la réalité et la connaissance, et que l'exercice de modélisation du réel est sans doute la démarche la plus importante et la plus difficile dans la démarche scientifique.

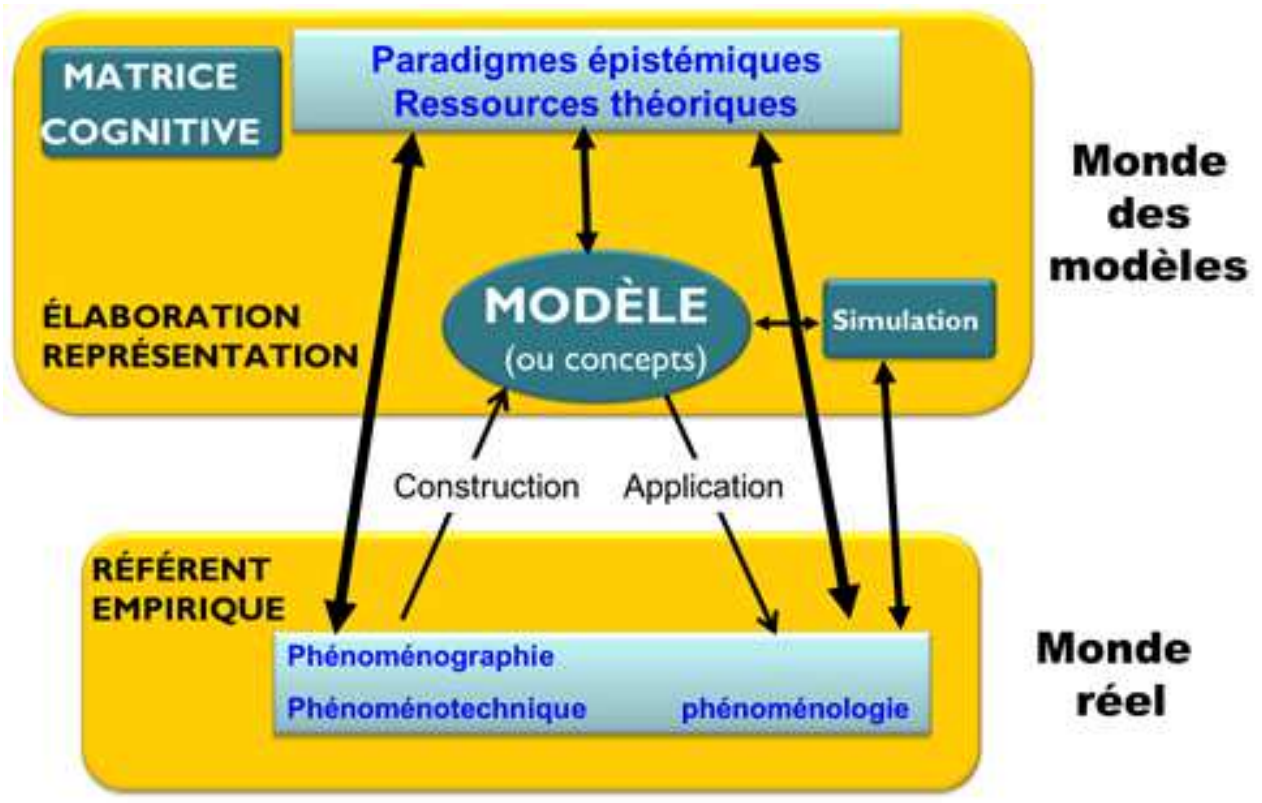

Fig. 1. L'activité de la modélisation dans la classe selon Martinand [7].

Selon ce schéma, Martinand décrit l'activité de la modélisation dans la classe, spécifiant la tâche de l'apprenant pendant l'apprentissage et décrit les événements se déroulant dans la classe du point de vue de l'enseignant et de la Physique. Elle consiste à établir des allers retours entre deux niveaux de modélisation ou entre deux mondes, "le monde réel" et "le monde des modèles" [7]. D'autres auteurs utilisent l'expression : la mise en relation ou les projections entre le "registre empirique" et le "registre du modèle" [8, 26, 27], et de repérer les liens entre eux dans une perspective dynamique de construction du savoir.

- Le registre empirique : représente les différents niveaux du réel construit par l'apprenant pour tenter de le comprendre. Il est constitué d'objets, de phénomènes et d'actions sur ces objets et phénomènes, en plus de procédés pris en compte soit par expérience directe, soit par évocation, et des premières connaissances d'ordre pratique qui leur sont associées comme: la phénoménographie (description première d'un phénomène impliquant déjà une construction conceptuelle antérieure), la phénoménotechnique (règles de montage, conditions de sécurité et savoir-faire instrumentaux) et la phénoménologie 
(description seconde de phénomènes en termes de concepts, modèles, théories partagées par une communauté de scientifiques).

- Le registre des modèles : représente le modèle qui permet d'interpréter et de représenter ce réel, comprenant des composantes sémantiques (une sémiographie commode pour représenter les éléments du modèle), syntaxiques (les relations qu'entretiennent les éléments du modèle entre eux liées à son mode de construction) et pragmatiques (qui permettent de questionner, se représenter, prévoir, inventer et expliquer le référent empirique).

À ces deux registres, Martinand en ajoute un troisième qui est "la matrice cognitive" appelée aussi "registre explicatif" [28]. Il comprend des paradigmes épistémiques, ainsi que des ressources théoriques et sémiotiques. La matrice cognitive représente les éléments théoriques qui appartiennent au système explicatif de l'apprenant. Ce registre comprend les types d'explications que l'apprenant est capable de mobiliser dans une situation donnée, ces types d'explication sont des règles générales extérieures à la discipline qui permettent à l'apprenant de construire ses propres propositions supposées vraies pour lui.

Ce schéma pour la modélisation guide les enseignants à choisir une démarche appropriée d'enseignement-apprentissage permettant de conduire leurs apprenants à acquérir différents modèles scientifiques. Il a été mis à l'épreuve dans plusieurs recherches, en particulier en didactique des sciences physiques, il a permis d'alimenter la réflexion sur le choix de stratégies d'enseignement et sur les registres à travailler pour choisir des démarches des modélisations des situations expérimentales.

On dispose maintenant d'un ensemble de données et d'analyses permettant de connaître la manière dont on peut enseigner et faire apprendre en concevant, en construisant, en manipulant et en adaptant des objets (réels ou virtuels) dans un but de modélisation scientifique. Dans cette approche, différents travaux de modélisation ont été réalisés, parmi lesquels les processus de modélisation mettant en œuvre les grandeurs fondamentales de la mécanique [29-31], la modélisation particulaire de la matière [32].

\section{Les points de vue des étudiants sur les modèles en sciences physiques}

\subsection{La construction du questionnaire}

Le questionnaire est le principal outil utilisé dans la présente étude pour la collecte des informations et des données sur le terrain. Ainsi, un questionnaire a été conçu pour un échantillon de 101 étudiants de la $3^{\text {ème }}$ année licence physique appartenant à trois universités algériennes différentes. Nous avons choisi des étudiants de trois échantillons issus de deux universités et une école (Tableau 1.).

Table 1. Répartition des étudiants questionnés.

\begin{tabular}{|c|c|c|c|}
\cline { 2 - 4 } \multicolumn{1}{c|}{} & $\begin{array}{c}\text { Université } \\
\text { Yahia Fares } \\
\text { Médéa }\end{array}$ & $\begin{array}{c}\text { Université } \\
\text { Saad Dahlab } \\
\text { Blida }\end{array}$ & $\begin{array}{c}\text { École Normale } \\
\text { Supérieure } \\
\text { Kouba }\end{array}$ \\
\hline Nombre d'étudiants & 40 & 32 & 29 \\
\hline Pourcentage & $39 \%$ & $32 \%$ & $29 \%$ \\
\hline
\end{tabular}

Nous évaluons que ce choix donne des résultats extrapolables pour la population concernée, étant donné que les universités adoptent le système L.M.D. (Licence, Master, Doctorat) et les écoles, le système classique, qui sont actuellement les deux systèmes en vigueur en Algérie. 
Le questionnaire a été établi afin d'étudier comment les étudiants conceptualisent les modèles et la modélisation. La variable exprimant cette étude comporte 4 indicateurs, chaque indicateur est explicité par 2 items (Annexe A) :

1- La prise en compte du concept de modèle;

2- L'identification du modèle et la cohérence de sa perception;

3- La perception de la nature hypothétique et modifiable du modèle;

4- La distinction entre modèle et réalité.

En effet, nous nous appuyons sur l'expérience vécue par ces acteurs durant leur carrière académique et sur leurs perceptions du concept de modèle tel qu'il a était enseigné même si implicitement cela a été fait pour décrire et analyser le dispositif mis en place. Nous nous sommes tenus dans la rédaction des items de notre questionnaire à utiliser un vocabulaire le plus familier aux répondants avec des expressions aussi courtes que possible. Nous avons préféré des questions semi-fermées. Une étape a suivi cette construction qui consiste à traduire les items du questionnaire en langue arabe pour certains étudiants pour ne laisser aucune barrière linguistique pouvant influencer la compréhension des expressions par ces acteurs et, par suite, fournir des réponses non intentionnelles.

Les étudiants n'avaient reçu aucun enseignement particulier sur les modèles scientifiques en sciences, leurs réponses reflètent donc leur compréhension basée sur le programme scientifique général qu'ils ont étudié. Nous notons que les étudiants universitaires de l'École Normale Supérieure ont suivi des cours dans les domaines de l'épistémologie et de la didactique, leur donnant un certain avantage dans leurs réponses, ce critère sera pris en compte lors de l'analyse des données.

\subsection{Résultats et discussion de questionnaire}

Afin d'analyser et d'interpréter les informations recueillies sur le terrain à travers le questionnaire, nous avons utilisés le logiciel SPSS (disponible en open source sur le site de IBM SPSS). Ce dernier nous a permis d'appliquer les statistiques descriptives sur notre échantillon et de réaliser des tests statistiques indispensables à notre analyse.

\subsubsection{L'indicateur relatif à la prise en compte du concept de modèle}

Le but de l'item 1.a est de savoir s'il existe une corrélation primaire entre l'étudiant et le concept de modèle, s'ils doivent être capables de prendre conscience des modèles impliqués dans une situation problème étudiée. Le tableau 2 et les figures 2-a et 2-b présentent les résultats concernant les items de l'indicateur relatif à la prise en compte du concept de modèle.

Dans cette étape, et en utilisant le logiciel SPSS, on a procédé de la manière suivante :

a) La codification et la saisie et des données.

Définir les variables et codifier les réponses en attribuant des valeurs numériques aux différents choix comme suit :

Pour l'item 1.a : Je ne sais pas $=1$; Jamais $=2$; Rarement $=3$; Souvent $=4$

Pour l'item 1.b : Je ne sais pas $=1$; Non $=2$; Oui $=3$

b) Réalisation d'une analyse descriptive des données.

Choisir d'obtenir des informations sous forme des tableaux (Fréquence (effective), pourcentage, moyenne et écart type).

La moyenne $\bar{X}$ d'une variable métrique dans différents sous-groupes mesure la tendance centrale. Elle permet de déterminer une valeur «typique» ou centrale autour de laquelle des données ont tendance à se rassembler. Elle est donnée par la relation: 


$$
\bar{X}=\frac{\sum n_{i} x_{i}}{N}
$$

L'écart type $\sigma$ permet de mesurer comment les données se dispersent par rapport à la moyenne. Il est donné par la relation:

$$
\sigma=\sqrt{V} \quad \text { avec } \quad V=\frac{\sum n_{i} x_{i}^{2}}{N}-\bar{X}^{2}
$$

La moyenne pour l'item 1.a possède une valeur très proche de 3 avec un écart type allant de 0.55 à 0.99 . Ces résultats montrent, d'une part, l'homogénéité des résultats obtenus dans les trois universités et de l'autre, que le terme modèle existe "rarement" dans la terminologie scientifique de la plus-part des étudiants.

La moyenne pour l'item 1.b a une valeur très proche de 2 avec une dispersion (écart-type) des valeurs autour d'elle variant entre 0.75 et 0.88 . Ces résultats montrent que la majorité des étudiants déclarent qu'ils ignorent la signification du terme modèle.

\begin{tabular}{|c|c|c|c|c|c|c|c|}
\hline \multicolumn{8}{|c|}{ Item 1.a : Au cours de vos études, avez-vous entendu couramment le terme "modèle" ? } \\
\hline & & Moyenne & Ecart type & Souvent & Rarement & Jamais & Je ne sais pas \\
\hline Médéa & $\mathrm{N}=40$ & 3,05 & 0,552 & $15 \%$ & $78 \%$ & $5 \%$ & $3 \%$ \\
\hline Blida & $\mathrm{N}=32$ & 2,94 & 0,716 & $19 \%$ & $59 \%$ & $19 \%$ & $3 \%$ \\
\hline Kouba & $\mathrm{N}=29$ & 3,07 & 0,998 & $38 \%$ & $45 \%$ & $3 \%$ & $14 \%$ \\
\hline Tout & $\mathrm{N}=101$ & 3,02 & 0,748 & $23 \%$ & $62 \%$ & $9 \%$ & $6 \%$ \\
\hline \multicolumn{8}{|c|}{ Item 1.b : Connaissez-vous la signification du terme : "modèle" ? } \\
\hline & & Moyenne & Ecart type & Oui & Non & \multicolumn{2}{|c|}{ Je ne sais pas } \\
\hline Médéa & $\mathrm{N}=40$ & 1,73 & 0,751 & $25 \%$ & $58 \%$ & \multicolumn{2}{|r|}{$17 \%$} \\
\hline Blida & $\mathrm{N}=32$ & 1,84 & 0,884 & $41 \%$ & $44 \%$ & \multicolumn{2}{|r|}{$16 \%$} \\
\hline Kouba & $\mathrm{N}=29$ & 1,76 & 0,689 & $38 \%$ & $41 \%$ & \multicolumn{2}{|r|}{$21 \%$} \\
\hline Tout & $\mathrm{N}=101$ & 1,77 & 0,773 & $34 \%$ & $49 \%$ & \multicolumn{2}{|r|}{$17 \%$} \\
\hline
\end{tabular}

Table 2. La prise en compte du concept de modèle.

Pour l'item 1.a, selon $62 \%$ des étudiants questionnés, le terme modèle est rarement mentionné par les enseignants alors que $23 \%$ pensent que leurs enseignants citent souvent le mot modèle en classe. Pour l'item 1.b, $66 \%(49+17) \%$ des étudiants ignorent la signification du concept de modèle, tandis que seulement $34 \%$ affirment comprendre le sens du terme modèle. 


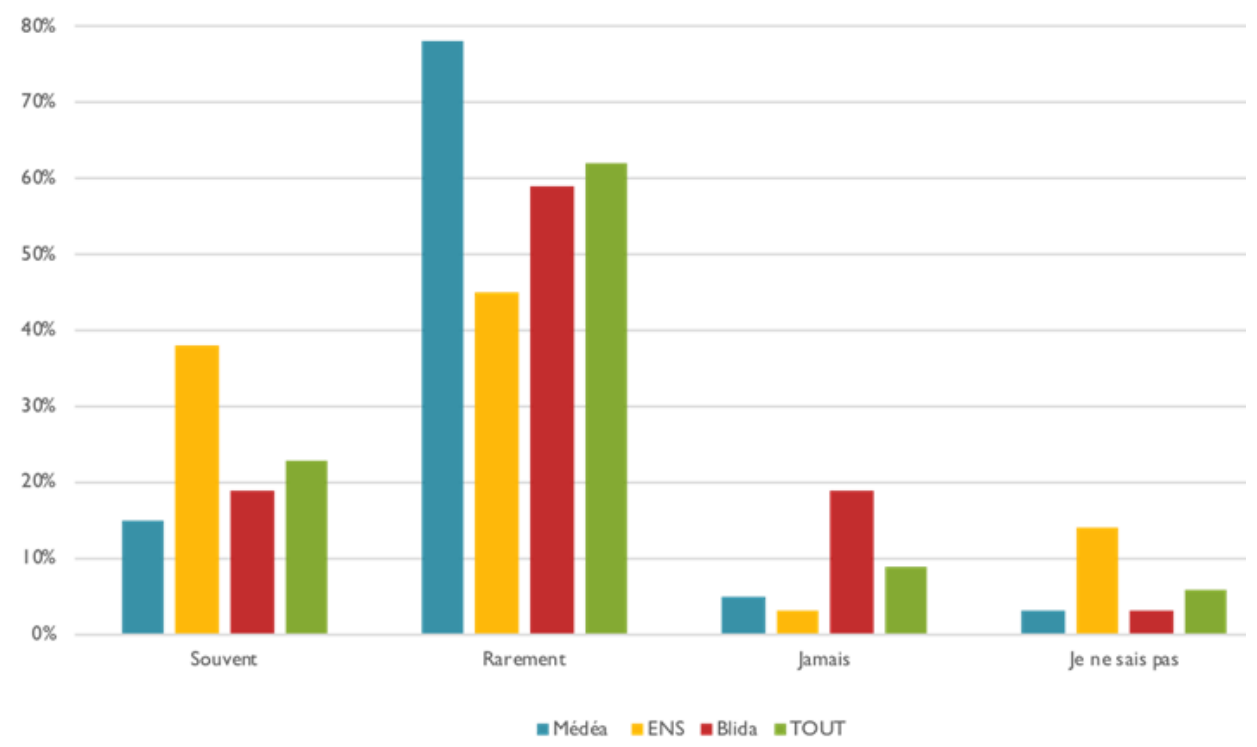

Fig. 2-a. La prise en compte du concept de modèle.

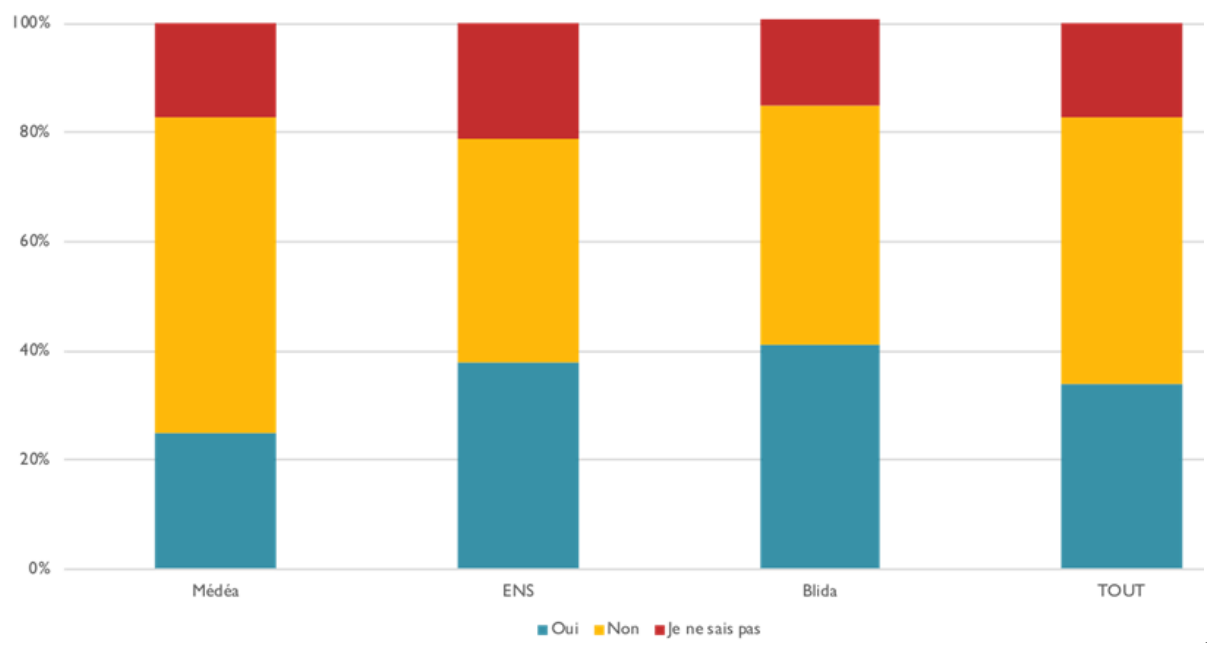

2-b. Taux de compréhension de sens du terme modèle.

Fig.

Le tableau 3 donne les exemples cités par les étudiants comme étant des modèles scientifiques. 
Table 3. Les modèles cités par les étudiants comme exemples.

\begin{tabular}{|l|c|c|c|c|}
\cline { 2 - 5 } \multicolumn{1}{c|}{} & \multicolumn{4}{c|}{ Fréquence } \\
\hline \multicolumn{1}{c|}{ Modèles scientifiques } & Médéa & Blida & Kouba & Total \\
\hline Modèle de Bohr & 15 & 11 & 16 & 42 \\
\hline Modèle de Rutherford & 8 & 4 & 5 & 17 \\
\hline Modèle de Lewis & 7 & 4 & 11 & 22 \\
\hline Modèle de champ & & & 1 & 1 \\
\hline Modèle mécanique & & & 2 & 2 \\
\hline Modèle du big-bang & & 1 & 1 & 2 \\
\hline Lois physiques & & 1 & 2 & 3 \\
\hline Modèle du gaz parfait & 3 & & & 3 \\
\hline Modèle standard & 1 & & 1 & 2 \\
\hline
\end{tabular}

Les modèles de Bohr, de Rutherford et de Lewis sont les plus cités par les étudiants des trois universités, en raison du fait que ces modèles sont explicitement introduits dans le programme d'études en tant que tels. En revanche, les modèles comme champs, lois, concepts, relation entre ces concepts, analogies, données d'expériences, formalisme mathématique... ne sont pas conçus comme des modèles. Ces résultats concernant le modèle en physique sont assez proches de ceux obtenus par Oldache [33] et Pelissier et Venturini [34] : selon eux, les représentations des étudiants questionnés relèvent de l'épistémologie moderne et la notion de modèle en elle-même est assez moderne.

\subsubsection{L'indicateur relatif à l'identification du modèle et la cohérence de sa perception}

Dans cette situation, on propose aux étudiants 05 paires d'exemples identiques réparties en deux séries. Dans la première, les étudiants sont invités à identifier les exemples qui représentent pour eux des modèles, et dans la seconde, ceux qui ne le sont pas. Le but de cette situation est d'une part, savoir si les étudiants ont la compétence de repérer des modèles et de types de modèles, et de l'autre, voir si leurs choix sont cohérents.

Les résultats de l'indicateur relatif à l'identification du modèle et la cohérence de sa perception sont rassemblés dans l'annexes B et l'annexe C.

Les résultats montrent tout d'abord que, parmi les 5 exemples proposés aux étudiants afin de choisir le "modèle", un seul exemple a obtenu l'accord majoritaire des étudiants des 3 universités. Il s'agit de l'exemple Ec « Un dispositif permettant l'étude de la chute libre ». $71 \%$ de la totalité des étudiants ont choisi cet exemple comme modèle. Ce choix est cohérent avec l'exemple E'c qui a obtenu l'accord minoritaire des étudiants des 3 universités comme exemple qui ne représente pas un modèle. L'exemple E'c « Ensemble de boules permettant l'étude des chocs. » est considéré par $68 \%$ de la totalité des étudiants comme étant un modèle. Les deux exemples Ec et E'c représentent une reproduction physique, qui est une représentation réduite ou simplifiée d'un phénomène. Ces exemples choisis par une grande partie des étudiants ciblés sont des modèles figuratifs qui traduisent la situation sous forme de phénomènes concrets homothétiques, ils sont classés comme un niveau primaire de la perception d'un modèle.

L'analyse des résultats de chaque université à part nous donne un regard différent sur les éléments pouvant constituer un point de vue des étudiants sur les modèles de la physique. Ainsi $72 \%$ et $90 \%$ des étudiants de l'université Saad Dahlab de Blida (USDB) considèrent respectivement $\mathrm{Eb}$ et E'b comme des modèles. Cela montre que des éléments relevant du champ théorique comme le point matériel et le rayon lumineux sont alors repérés et pris en 
compte par ces étudiants comme modèles. En outre $52 \%$ et $66 \%$ des étudiants de L'ENS de Kouba (ENSK) considèrent respectivement les exemples Ee et E'e comme des modèles, un point de vue qui se rapproche à celui des étudiants de l'université Yahia Fares de Médéa (UYFM) avec des taux respectifs de $57 \%$ et $55 \%$. Cela montre que ces étudiants disposent d'un critère comme loi et relation mathématique pour identifier un modèle. La paire d'exemples Ed et E'd a été choisie par les étudiants de (USDB), leurs pourcentages sont respectivement $63 \%$ et $78 \%$, ces résultats montrent que ces étudiants définissent le modèle comme étant un diagramme, schéma, image, graphique ou autre de même nature.

Cette divergence entre les choix des étudiants peut s'expliquer par la compréhension partielle du concept de modèle, de sa construction et des attributs permettant de l'identifier. Nos résultats sont conformes à ceux obtenus par des travaux antérieurs [35-38] qui ont montrés que les élèves et les enseignants du secondaire ont en général une compréhension épistémologique limitée de la notion de modèle et de la modélisation.

On constate qu'une proportion non négligeable de la totalité des étudiants $(52 \%$, $63 \%, 56 \%)$ ne considèrent pas respectivement les exemples Eb, Ed, et Ee comme modèles (annexes B), tandis qu'un pourcentage important $(55 \%, 75 \%, 58 \%)$ des étudiants considèrent respectivement les exemples E'b, E'd et E'e comme modèles (annexes C). L'exemple Ed est exclu par $80 \%$ des étudiants (UYFM) comme étant un modèle alors que son équivalent E'd est choisi par $80 \%$ en tant que modèle par les mêmes étudiants. La majorité des étudiants appliquent donc un argument pour choisir un exemple comme modèle et l'exclut pour un autre de même nature. Ces résultats montrent que les étudiants trouvent des difficultés à discerner les modèles théoriques ou "abstraits" qui ne renvoient pas à des aspects directement observables du réel. Les résultats montrent également qu'un nombre important d'étudiants n'ont pas la capacité de discerner parfaitement ce qu'est un modèle et ne possèdent pas les critères scientifiques d'un choix de modèle.

Le tableau 4, donne la fréquence (l'effectif) et les pourcentages calculés par le logiciel SPSS de toutes les réponses cohérentes données par les étudiants. Tout d'abord, on a calculé la moyenne de tous les choix cohérents pour une paire de réponse ( 5 possibilités), puis deux paires (10 possibilités), trois paires (10 possibilités), et quatre paires cohérentes (5 possibilités), et enfin la valeur de la fréquence (l'effectif) et le pourcentage des cinq paires de réponses (1 possibilité).

Les résultats collectés auprès des étudiants (Fig. 3.) montrent qu'une moyenne de $62 \%$ des répondants a donné un choix d'une paire cohérente concernant l'identification de modèle, alors que seulement $33 \%$ d'eux sont capables de donner deux réponses cohérentes. Le taux des répondants décroit rapidement vers 13\% lorsqu'il s'agit de 04 réponses. Enfin seulement $9 \%$ des étudiants ciblés sont capables à donner 05 réponses cohérentes lors de désignation des modèles. Ces résultats montrent en effet une forte instabilité et incohérence des choix des étudiants. La stabilité apparait tout d'abord lorsqu'il s'agit d'un premier niveau qui correspond aux étudiants qui pensent que les modèles sont des objets matériels construits pour copier la réalité (la paire Ed et E'd). Mais au second niveau où le modèle est abstrait ou fait partie du monde théorique, l'instabilité et l'incohérence augmentent, ce qui explique une vision incomplète de la nature des modèles et l'absence d'une référence permettant de les différencier. Le troisième niveau correspondant à celui accepté par les experts est loin d'être acquis. 
Table 4. Résultats de la cohérence de la perception du modèle.

\begin{tabular}{|c|c|c|c|c|c|c|c|c|c|c|c|}
\hline & & & équ & $(\mathbf{E} f$ & ctif) & & & & ourcen & & \\
\hline $\begin{array}{r}\text { Nombr } \\
\text { de } r \\
\text { coh }\end{array}$ & $\begin{array}{l}\text { de paires } \\
\text { onses } \\
\text { entes }\end{array}$ & \begin{tabular}{l}
$\Theta$ \\
\multirow{0}{*}{} \\
0
\end{tabular} & $\begin{array}{l}\text { N } \\
\text { D. } \\
\text { : }\end{array}$ & 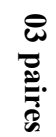 & 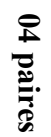 & 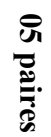 & 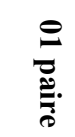 & 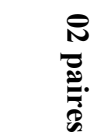 & 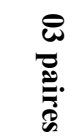 & 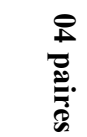 & 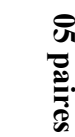 \\
\hline Médéa & $\mathrm{N}=40$ & 24 & 11 & 6 & 3 & 3 & $60 \%$ & $27,5 \%$ & $15 \%$ & $7,5 \%$ & $7,5 \%$ \\
\hline Blida & $\mathrm{N}=32$ & 19 & 9 & 9 & 6 & 3 & $59 \%$ & $28 \%$ & $28 \%$ & $19 \%$ & $9 \%$ \\
\hline Kouba & $\mathrm{N}=29$ & 20 & 13 & 6 & 4 & 3 & $69 \%$ & $45 \%$ & $21 \%$ & $14 \%$ & $10 \%$ \\
\hline Tout & $\mathrm{N}=101$ & 63 & 33 & 21 & 13 & 9 & $62 \%$ & $33 \%$ & $21 \%$ & $13 \%$ & $9 \%$ \\
\hline
\end{tabular}

\section{Le taux de choix des paires cohérentes}

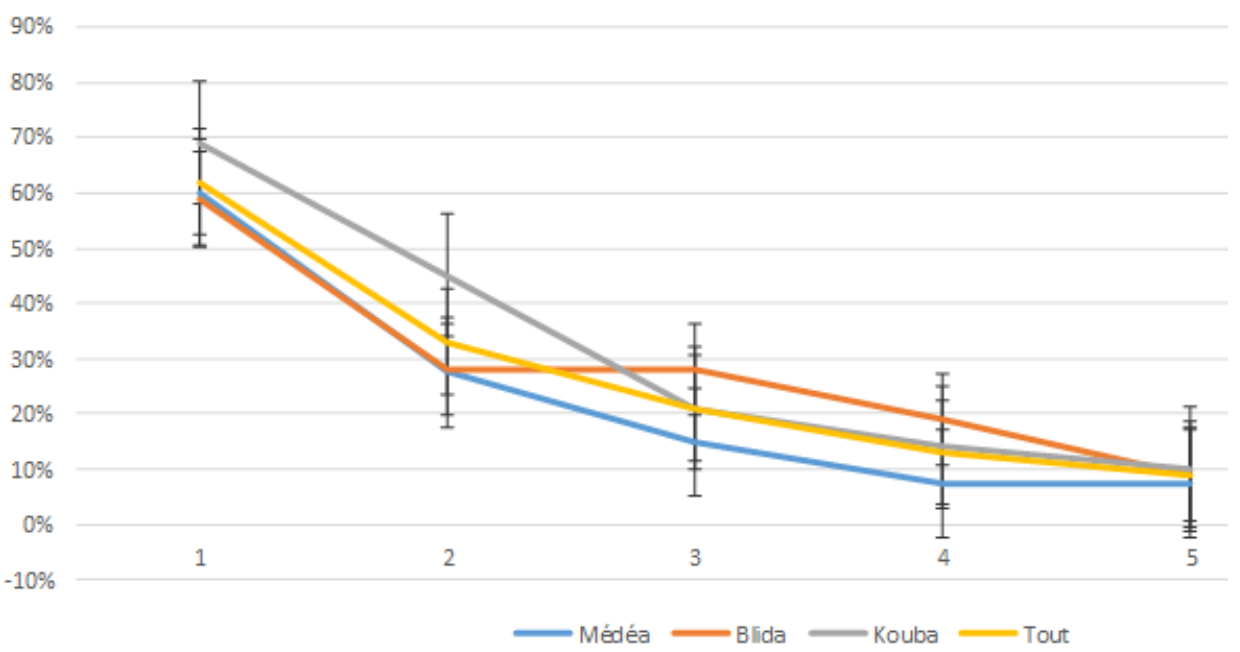

Fig. 3. La cohérence des réponses des étudiants.

\subsubsection{L'indicateur relatif à la nature hypothétique et modifiable du modèle}

La modification du modèle est une opportunité d'obtenir plus d'informations. Dans l'activité de modélisation, le changement de modèle implique nécessairement un changement de compréhension du monde réel. C'est pourquoi une des caractéristiques essentielles dans la construction, l'adaptation et l'utilisation de modèles en classe est la nature hypothétique, provisoire et transformable des modèles. L'objectif des items 3.a et 3.b est de savoir si les étudiants perçoivent cette caractéristique. Le tableau 5 donne les résultats de leurs réponses. 
Table 5. Résultats des items 3.a et 3.b : La nature hypothétique et modifiable du modèle.

\begin{tabular}{|l|c|c|c|}
\hline \multirow{2}{*}{ Item 3.a } & Université & Oui & Non \\
\hline \multirow{2}{*}{$\begin{array}{l}\text { Pouvons-nous représenter un } \\
\text { phénomène avec différents } \\
\text { modèles }\end{array}$} & Médéa & $47,5 \%$ & $52,5 \%$ \\
\cline { 2 - 4 } & Blida & $53 \%$ & $47 \%$ \\
\cline { 2 - 4 } & Kouba & $45 \%$ & $55 \%$ \\
\cline { 2 - 4 } Item 3.b & Total & $48,5 \%$ & $51,5 \%$ \\
\hline \multirow{2}{*}{$\begin{array}{l}\text { La lumière possède deux } \\
\text { aspects différents : ondulatoire } \\
\text { et corpusculaire. On dit les deux } \\
\text { aspects sont des : }\end{array}$} & Université & $\begin{array}{c}\text { Réalité } \\
\text { physique }\end{array}$ & $\begin{array}{c}\text { Modèles } \\
\text { physique }\end{array}$ \\
\cline { 2 - 4 } & Médéa & $55 \%$ & $45 \%$ \\
\cline { 2 - 4 } & Blida & $81 \%$ & $19 \%$ \\
\cline { 2 - 4 } & Kouba & $72 \%$ & $28 \%$ \\
\hline
\end{tabular}

Ces résultats montrent que la majorité des étudiants ne perçoivent pas la nature hypothétique, provisoire et transformable des modèles. $51,5 \%$ des étudiants affirment qu'un phénomène est représenté par un seul modèle et $68 \%$ d'eux considèrent les deux aspects de la lumière comme réalités physiques et non pas comme des modèles différents. La nécessité de modèles multiples n'est pas reconnue par la plupart des étudiants, ils ignorent donc l'utilité de disposer de plusieurs modèles pour montrer différentes vues de la même entité.

Les résultats ont également montré que sur l'effectif de 101 étudiants interrogés, seuls 16 étudiants $(16 \%)$ ont donné des réponses d'experts, affirmant l'existence des modèles multiples pour un seul phénomène (Item 3.a) et considérant les deux aspects de la lumières en tant que des modèles (Item 3.b). 36 étudiants (36\%) ont donné des réponses complètement opposées aux réponses d'experts, alors que, 49 étudiants (48\%) ont donné des réponses incohérentes.

\subsubsection{L'indicateur relatif à la différenciation entre modèle et réalité}

La distinction entre modèle et réalité est au cœur de la compréhension épistémologique de la notion de modèle, et la confusion modèle/réalité peut être un obstacle didactique à l'accès des apprenants à la construction des modèles et à la pratique d'une activité de modélisation.

Des travaux antérieurs montrent que les enseignants et leurs apprenants ont des conceptions épistémologiques généralement réalistes et un empirisme que l'on peut dire " naïf » et inductiviste [39]. Ainsi, les travaux d'Oldache auprès d'étudiants de l'ENS-Kouba montrent que leurs conceptions en ce qui concerne les concepts fondamentaux de la physique de base, tels que, la particule isolée, le point matériel, l'atome, la particule ultime, le champ et la force, relèvent d'une épistémologie réaliste [33]. L'auteur affirme que ses résultats obtenus avec les étudiants algériens concordent largement avec ceux obtenus avec des étudiants américains en citant une étude menée par Baily sur la fonction d'onde [40].

L'objectif des items 4.a et 4.b est de savoir si les étudiants font la distinction entre modèle et réalité. S'ils ont une perception réaliste de la science, cela pourrait-il être la source d'un conflit épistémique entre modèle et réalité. Le tableau 6 donne les résultats de leurs réponses. 
Table 6. Résultats des items 4.a et 4.b : La différenciation entre modèle et réalité

\begin{tabular}{|l|c|c|c|}
\hline \multirow{2}{*}{ Item 4.a } & Université & Modèles & Réalité \\
\hline \multirow{2}{*}{$\begin{array}{l}\text { Un point matériel, un rayon } \\
\text { lumineux, une force, un champ } \\
\text { électrique ou magnétique, une } \\
\text { vitesse sont considérés pour } \\
\text { vous comme : }\end{array}$} & Médéa & $2,5 \%$ & $97,5 \%$ \\
\cline { 2 - 4 } & Blida & $6 \%$ & $94 \%$ \\
\cline { 2 - 4 } & Touba & $17 \%$ & $83 \%$ \\
\hline \multirow{2}{*}{$\begin{array}{l}\text { Les lois physiques et les } \\
\text { relations mathématiques sont } \\
\text { considéré pour vous comme : }\end{array}$} & Université & $8 \%$ & $92 \%$ \\
\cline { 2 - 4 } & Médéa & $42,5 \%$ & Redèles \\
\cline { 2 - 4 } & Blida & $16 \%$ & $87,5 \%$ \\
\cline { 2 - 4 } & Kouba & $31 \%$ & $69 \%$ \\
\hline
\end{tabular}

Le tableau 6 et les figures 4-a et 4-b montrent qu'une grande majorité des étudiants des 3 universités $(92 \%)$ considèrent le point matériel, le rayon lumineux, la force, le champ électrique ou magnétique et la vitesse comme une réalité alors que seulement (8\%) perçoivent ces concepts comme des modèles. (69\%) des étudiants excluent les lois et les relations mathématiques des modèles.

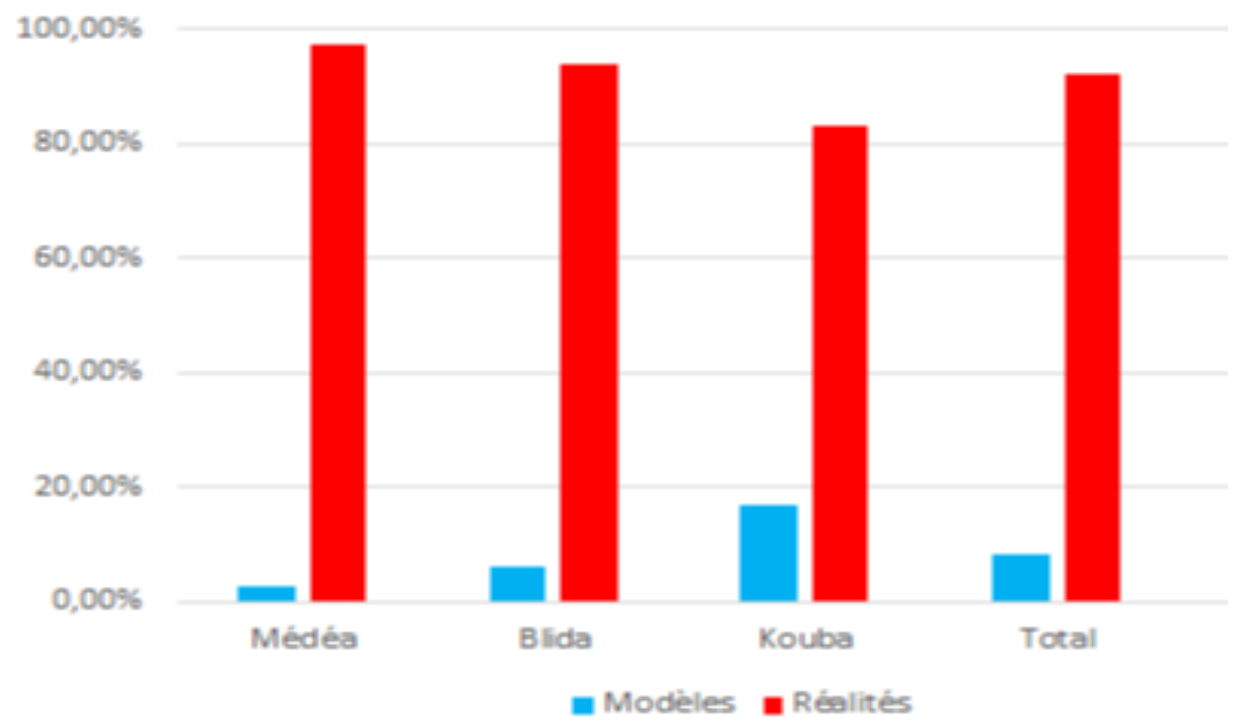

Fig. 4-a. Résultats de l'item 4.a. La différenciation entre modèle et réalité. 


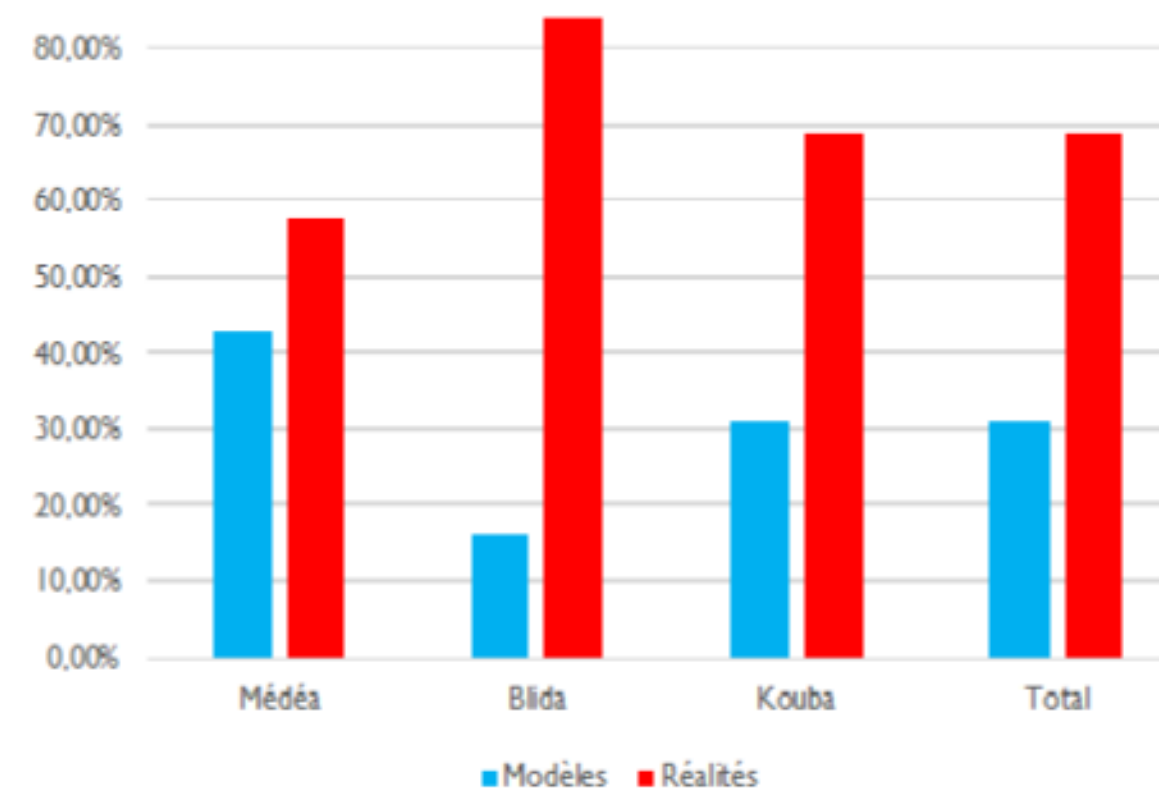

Fig. 4-b. Résultats de l'item 4.b. La différenciation entre modèle et réalité.

Ces résultats montrent que la plupart des étudiants ont la conception que les modèles appris en classe sont la réalité des phénomènes. Ils ne perçoivent pas la différence entre réalité et modèle. Ce point de vue découle probablement du réalisme naïf qu'une majorité d'entre eux manifeste à l'égard de la conception de la science. Halbwaches pense que la multitude des modèles descriptifs étudiés en classe seraient à l'origine de la confusion entre modèle et réalité [41].

Ces résultats sont identiques à ceux obtenus par l'équipe de Grosslight [23], son étude montre que les étudiants avaient souvent eu des conceptions qui correspondaient à une épistémologie du "réaliste". Ainsi, ils sont plus susceptibles de considérer les modèles comme des copies physiques de la réalité qui incarnent différentes perspectives spatiotemporelles.

Pour éviter de confondre modèle et réalité, il est important que les étudiants construisent eux même le modèle, ce qui nécessite d'établir des liens entre le modèle et la réalité modélisée. Cette activité de modélisation leur permet de comprendre que le modèle est un outil intermédiaire entre réalité et connaissance. Cette activité les aidera à faire le lien entre ces deux mondes.

Des travaux menés par plusieurs auteurs dans ce sens proposent différentes stratégies didactiques susceptibles de faciliter aux apprenants la distinction entre modèle et réalité. Certains [42], proposent l'apprentissage progressif, actif et épistémologique de la notion de modèle. Il s'agit d'en définir ses caractères et ses conditions de validité, en insistant dès les premiers niveaux scolaires de classe sur l'exigence de différenciation entre le modèle et le réel. D'autres conseillent de placer les enseignants dans les meilleures conditions pour enseigner cette différence, avoir une bonne connaissance de la "Nature de la Science" [43], avoir une solide compréhension épistémologique de la notion de modèle, qui revêt une importance primordiale dans la conception et la conduite des situations d'enseignement [44]. D'autres suggèrent de conduire l'apprenant à différencier les modèles de la réalité empirique observable et de la réalité théorique scientifique, c'est à dire ceux qui représentent une réalité visible de ceux qui représentent une réalité invisible [9]. Morge et Doly [5] recommandent à 
l'enseignant de s'appuyer sur des exemples qui favorisent la réflexion épistémologique qui permet à l'apprenant de différencier modèle et réalité. Ces exemples doivent :

1- Permettre de rompre avec la relation biunivoque modèle-réalité.

2- Permettre de présenter un écart important avec la réalité.

3- Permettre aux apprenants de modéliser des objets perceptibles.

Trois conditions didactiques semblent nécessaires à ces auteurs pour l'enseignement de la modélisation permettant aux apprenants de faire la différence entre modèle et réalité :

1- L'objet modélisé doit être directement perceptible.

2- L'objet doit pouvoir être modélisé de différentes manières.

3- L'objet et son modèle ne doivent pas être dans un rapport descriptif.

\section{Conclusion et perspectives}

Le mot modèle est absent dans la terminologie scientifique de la plupart des étudiants malgré son utilisation fréquente par leurs enseignants, ce qui pose un problème de contrat didactique. S'il existe chez certains, la majorité conçoivent les modèles comme outil de représentation, de simplification et de matérialisation, ils sont reconnus pour servir la visualisation et l'explication, ce qui reflète une compréhension simple et limitée des modèles, qui est moins élaborée que les conceptions scientifiques.

Dans notre étude qui visait le développement structuré d'un outil didactique, nous avons constaté que les étudiants observés ne mettent pas en relation les faits (le concret qu'on veut expliquer) et les théories (avec quoi on veut expliquer), car les activités d'enseignement actuelles ne répondent que faiblement à la construction de modèle, un concept central et fondamental de la science, qui se trouve à l'exacte frontière entre fait et idée ou matérialité et théorie. Les apprenants doivent comprendre que l'activité essentielle d'un scientifique réside dans la mise en relation entre les deux via des modèles.

De même, l'instabilité et l'incohérence des choix des étudiants indiquent l'absence d'une part, des critères leurs permettant la classification des modèles et, d'autre part, d'une méthode permettant la distinction entre les champs empiriques des champs théoriques, et la prise en compte de modèle en tant qu'objet intermédiaire.

Les résultats de cette enquête montrent aussi que pour la majorité des étudiants, les relations mathématiques sont exclues des modèles scientifiques. La nature changeante des modèles scientifiques, n'est pas reconnue par la plupart des étudiants. Ainsi la distinction entre modèle et réalité, qui est au centre de la notion de modèle, est bien éloignée de la perception des apprenants.

De nombreux étudiants n'appréhendent donc pas pleinement les modèles scientifiques, ce qui indique le manque d'opportunité d'utiliser efficacement les modèles et leurs applications en classe, ou les enseignants ne se placent pas généralement dans les meilleures conditions pour clarifier la notion de modèle. Nos résultats montrent également que les notions relatives aux modèles et à la modélisation peuvent être accessibles aux étudiants. En effet, il apparaîtrait nécessaire de s'appuyer sur de telles études pour articuler un cadre didactique et un cadre épistémologique afin de construire des faits didactiques liés à l'enseignement du concept de modèle. D'autre part, des sujets tels que l'histoire des sciences, l'histoire de la physique, l'épistémologie et la didactique dans les programmes de formation licence et master, ainsi que l'enseignement de la notion de modèle de manière explicite peuvent combler ces lacunes et permettent de placer les étudiants dans des conditions favorables à la construction de la notion de modèle et à l'apprentissage de la modélisation.

Ces résultats de recherche pourraient être pris en compte dans la conception des programmes visant à surmonter ces problèmes en accompagnant l'étudiant pour lui permettre 
de construire la notion de modèles et de comprendre leurs fonctions et leurs évolutions et d'identifier l'activité de la modélisation dans la démarche scientifique.

\section{Remerciements}

Je remercie vivement tous les membres du comité scientifique et d'organisation de la $3^{\text {ème }}$ édition du colloque international CIFEM'2020, je tiens à remercie particulièrement les évaluateurs de la première et de la deuxième phase pour leurs lectures et corrections et leurs précieux commentaires. J'adresse également un merci spécial à mon directeur de laboratoire (LPTEA) le Pr Zitouni Hannachi pour son soutien et ses recommandations judicieuses.

We thank the General Directorate of Scientific Research and Technological Development (DGRSDT), Algiers, Algeria for the financial support.

\section{Références}

1. M. Janosz, L'abandon scolaire chez les adolescents : perspective nord-américaine. Enjeux, 105-127 (2000)

2. M. Melhaoui, L. El hafid, B. Amamou \& A. Hammouti, L'échec à l'université Marocaine : bilan des principales causes et attentes de la pédagogie universitaire. Dans AIPU. Actes du $21^{\text {ème }}$ Congrès international de l'AIPU, 3-7 Mai 2004, pp. 22-23, (Publication de l'université Cadi Ayyad, Marrakech, Maroc, 2004)

3. M. Jones, C. Eick, Implementing inquiry kit curriculum: Obstacles, adaptations, and practical knowledge development in two middle school science teachers. Science Education, 3, 91, 492-513 (2007)

4. N. Hulin, L'histoire des sciences et son inscription institutionnelle, Dans J. Rosmorduc (dir.) Histoire des sciences et des techniques, 39-69 (CRDP, Rennes, 1997)

5. L. Morge, A.-M Doly, L'enseignement de notion de modèle : quels modèles pour faire comprendre la distinction entre modèle et réalité ? Revue de recherches en éducation, 52, 149-175 (2013)

6. J.-L. Martinand, Enseignement et apprentissage de la modélisation en sciences (INRP, Paris, 1992)

7. J.-L. Martinand, Introduction à la modélisation. Communication présentée à la conférence Actes du séminaire de didactique des disciplines technologiques, 126-138, Cachan, Paris (1994)

8. A. Tiberghien, Modeling as a basis for analyzing teaching-learning situations. Learning and instructions, 4, 71-87 (1994)

9. G. Robardet, J. C. Guillaud, Éléments de didactique des sciences physiques. De la recherche à la pratique : théorie, modèle, conceptions et raisonnement spontané (PUF, Paris, 1997)

10. I. A. Halloun, Modeling theory in science education (Kluwer Academic Publishers, Dordrecht, Holland, 2004)

11. I. Damska, Le concept de modèle et son rôle dans les sciences. Revue de synthèse, 3951 (1959)

12. G. Canguilhem, Modèles et analogies dans la découverte en biologie, études d'histoire et de philosophie des sciences (Vrin, Paris, 1968)

13. M. Bunge, Method, model, and matter (Dordrecht, NL, Reidel., 1973)

14. B. Walliser, Système et modèles. (Seuil, Paris, 1977) 
15. S. Bachelard, Quelques aspects historiques des notions de modèle et de justification des modèles. In P. Delattre \& M. Thellier (Eds.), Elaboration et justification des modèles. 09-20 (Maloine, Paris, 1979)

16. M. Suarez, Theories, models, and representations. In L. Magnani, N. J. Nersessian \& P. Thagard (Eds.), Model-based reasoning in scientific discovery. 75-99 (Kluwer Academic/Plenum Press, New York, 1999)

17. D. M. Bailer-jones, Scientists' thoughts on scientific models. Perspectives on Science, 3, 10, 275-301 (2002)

18. T.Van Der valk, J. H. Van Driel \& W. De Vos, Common characteristics of models in present-day scientific practice. Research in Science Education, 4, 37, 469-488 (2007)

19. U. Besson, Calculating and understanding: Formal models and causal explanations in science, common reasoning and physics teaching. Science \& Education, 3, 19, 225-257 (2010)

20. A. G. Harrison, Thinking and Working Scientifically: The role of Analogical and Mental Models. Papier présenté à la conférence annuelle: meeting of the Australian Association for Research in Education 2-6 December (Fremantle, 2001)

21. J. Clement, Model based learning as a key research area for science education. International Journal of Science Education, 9, 22, 1041-1053 (2000)

22. J. K. Gilbert, C. Boulter, Developing models in science education (Kluwer Academic Publishers, Dordrecht, Holland, 2000)

23. L. Grosslight, C. Unger, E. Jay, Understanding models and their use in science: Conceptions of middle and high school students and experts. Journal of Research in Science Teaching, 9, 28, vol., 799-822 (1991)

24. G. D. Chittleborough, D. F. Treagust, T. L. Mamiala, \& M. Mocerino, Students' perceptions of the role of models in the process of science and in the process of learning. Research in Science \& Technological Education, 2, 23, 195-212 (2005)

25. D. F. Treagust, G. D. Chittleborough \& T. L. Mamiala, Students' understanding of the role of scientific models in learning science. International Journal of Science Education, 24, 357-368 (2002)

26. K. Becu-Robinault, Modelling activities of students during a traditional labwork. In D. Psillos \& H. Niedderer (Eds.), Teaching and learning in the science laboratory (Kluwer Academic Publishers, Dordrecht, Holland, 2002)

27. C. Buty, A. Tiberghien \& J.-F. Le Maréchal, Learning hypotheses and an associated tool to design and to analyse teaching-learning sequences. International Journal of Science Education 26, 579-604 (2004)

28. C. Orange, Problèmes et modélisation en biologie (PUF, Paris, 1997)

29. A. Weil-Barais, et G. Lemeignan, Apprentissage de concepts en mécanique et modélisation de situations expérimentales. European Journal of Psychology of Education, 4, 5, 391-415 (1990)

30. G. Lemeignan, A. Weil-Barais, Gestion d'activités de modélisation en classe. Aster,7, 121-141 (1993)

31. G. Lemeignan, A. Weil-Barais, Construire des concepts en Physique (Hachette, Paris, 1993)

32. C. Larcher, A. Chomat, Modélisation de la matière en cycle central du collège. Bulletin de l'union des Physiciens, 826, 1341-136 (2000) 
33. M. Oldache, Problématique de la relation entre le perçu et le réel en physique moderne et son impact sur l'enseignement. Thèse de Doctorat, ENS/Kouba, Département de Physique. (Alger, 2014)

34. L. Pelissier, P. Venturini, Analyse praxéologique de l'enseignement de l'épistémologie de la physique : le cas de la notion de modèle. Education \& Didactique, 10 (Presses Universitaires. Rennes, 2016)

35. S. W. Gilbert, Model building and a definition of science. Journal of Research in Science Teaching, 1, 28, 73-79 (1991)

36. J. H. Van Driel, N.Verloop, Teachers' knowledge of models and modelling in science. International Journal of Science Education, 11, 21, 1141-1153 (1999)

37. J. H. Van Driel, N.Verloop, Experienced teachers' knowledge of teaching and learning of models and modelling in science education. International Journal of Science Education, 12, 24, 1255-1272 (2002)

38. B. A. Crawford, M. J. Cullin, Supporting prospective teachers' conceptions of modeling in science. International Journal of Science Education, 11, 26, 1379-1401 (2004)

39. M. T. Guerra-Ramos, Teachers' Ideas about the Nature of Science: A Critical Analysis of Research Approaches and Their Contribution to Pedagogical Practice. Science \& Education 5, 21, 631-655 (2012)

40. C.R. Baily, Perpectives in Quantum Physics: Epistemological, Ontological and Pedegogical, an investigation into student and expert perspectives on the physical interpretation of quantum mechanics, with implications for modern physics instruction ( $\mathrm{PhD}$ thesis). University of Colorado at Boulder, Department of Physics (Boulder, 2011)

41. F. Halbwaches, La Pensée physique chez l'enfant et le savant. p.42 (Delachaux et Niestlé, 1974)

42. V. Munier, D. Passelaigue, Réflexions sur l'articulation entre didactique et épistémologie dans le domaine des grandeurs et mesures dans l'enseignement primaire et secondaire. Tréma, 38, 107-147 (2012)

43. F. Abd-el-khalick, N. Lederman, The influence of the history of science courses on students'views of the nature of science. Journal of Research in Science Teaching, 10, 37, 1057-1095 (2000)

44. R. G. Justi, J. Van Driel, The development of science teachers' knowledge on models and modelling: promoting, characterizing, and understanding the process. International Journal of Science Education 5, 27, 549-573 (2005) 


\section{ANNEXE A \\ QUESTIONNAIRE}

Ce questionnaire s'adresse aux étudiants de $3^{\text {ème }}$ année licence physique, il a pour objectif d'étudier comment les étudiants perçoivent la notion de modèle, leur position et la façon d'approprier ce concept. Nous vous prions de bien vouloir remplir ce questionnaire, en choisissant la bonne réponse.

1- Au cours de vos études, avez-vous entendu couramment le terme "modèle" ?

$\begin{array}{ll}\text { a- } & \text { Souvent } \\ \text { b- } & \text { Rarement } \\ \text { c- Jamais, } \\ \text { d- }\end{array}$

2- Connaissez-vous la signification du terme "modèle" ?

a- Oui.

b- Non

c- Je ne sais pas

Si oui citez des exemples:

3- Parmi ces cinq exemples, lequel ou lesquels représente(nt) un modèle ?

a- Une voiture se déplace sur un sol horizontal.

b- Un point matériel se déplace à vitesse constante.

c- Un dispositif permettant l'étude de la chute libre.

d- Diagramme de vitesse d'un mouvement rectiligne

e- La relation mathématique : $\vec{F}=m \vec{a}$

4- Parmi ces cinq exemples, lequel ou lesquels ne représente(nt) pas un modèle ?

a- Le soleil émet de la lumière.

b- La réflexion d'un rayon lumineux arrivant sur un dioptre.

c- Ensemble de boules permettant l'étude des chocs.

d- Schéma d'un solide suspendu à un ressort vertical.

e- L'équation horaire d'un mouvement rectiligne : $x=v t+x_{0}$

5- Pouvons-nous représenter un phénomène avec différents modèles ?

$\begin{array}{ll}\text { a- } & \text { Oui } \\ \text { b- } & \text { Non }\end{array}$

6- Un point matériel, un rayon lumineux, une force, un champ électrique ou magnétique, une vitesse sont considérés pour vous comme :

a- Modèles

b- Réalités

7- Les lois physiques et les relations mathématiques sont considéré pour vous comme :
a- Modèles
b- Réalités

8- La lumière possède deux aspects différents : ondulatoire et corpusculaire. On dit :

a- Les deux aspects sont des réalités physiques différentes.

b- Les deux aspects sont des modèles physiques différents. 


\section{ANNEXE B}

\section{Les résultats de l'indicateur relatif à l'identification du modèle et la cohérence de sa perception}

\section{Les exemples qui représentent des modèles}

\begin{tabular}{|c|c|c|c|c|c|c|}
\hline Item 2.a & \multicolumn{2}{|c|}{ Université } & Moyenne & Ecart type & Oui & Non \\
\hline \multirow{3}{*}{$\begin{array}{l}\text { Ea- Une voiture se déplace sur un sol } \\
\text { horizontal }\end{array}$} & \multicolumn{2}{|c|}{ Médéa } & 1,60 & 0,496 & $40 \%$ & $60 \%$ \\
\hline & \multicolumn{2}{|c|}{ Blida } & 1,44 & 0,504 & $56 \%$ & $44^{\circ}$ \\
\hline & \multicolumn{2}{|c|}{ Kouba } & 1,76 & 0,435 & $76 \%$ & 24 \\
\hline \multirow{3}{*}{$\begin{array}{l}\text { Eb- Un point matériel se déplace à vitesse } \\
\text { constante }\end{array}$} & \multicolumn{2}{|c|}{ Médéa } & 1,58 & 0,501 & $42 \%$ & $58 \%$ \\
\hline & \multicolumn{2}{|c|}{ Blida } & 1,28 & 0,457 & $72 \%$ & $28^{\circ}$ \\
\hline & \multicolumn{2}{|c|}{ Kouba } & 1,69 & 0,471 & $31 \%$ & $69 \%$ \\
\hline \multirow{3}{*}{$\begin{array}{l}\text { Ec- Un dispositif permettant l'étude de la } \\
\text { chute libre }\end{array}$} & \multicolumn{2}{|c|}{ Médéa } & 1,28 & 0,452 & $73 \%$ & 27 \\
\hline & \multicolumn{2}{|c|}{ Blida } & 1,31 & 0,471 & $69 \%$ & $31^{\circ}$ \\
\hline & \multicolumn{2}{|c|}{ Kouba } & 1,28 & 0,455 & $72 \%$ & $28^{\circ}$ \\
\hline \multirow{3}{*}{$\begin{array}{l}\text { Ed- Diagramme de vitesse d'un mouvement } \\
\text { rectiligne }\end{array}$} & \multicolumn{2}{|c|}{ Médéa } & 1,80 & 0,405 & $20 \%$ & $80 \%$ \\
\hline & \multicolumn{2}{|c|}{ Blida } & 1,38 & 0,492 & $63 \%$ & $37 \%$ \\
\hline & \multicolumn{2}{|c|}{ Kouba } & 1,69 & 0,471 & $31 \%$ & $69 \%$ \\
\hline \multirow[t]{3}{*}{ Ef- La relation mathématique $: \vec{F}=m \vec{a}$} & \multicolumn{2}{|c|}{ Médéa } & 1,55 & 0,504 & $57 \%$ & $43 \%$ \\
\hline & \multicolumn{2}{|c|}{ Blida } & 1,66 & 0,483 & $34 \%$ & $66 \%$ \\
\hline & \multicolumn{2}{|c|}{ Kouba } & 1,48 & 0,509 & $52 \%$ & 48 \\
\hline \multicolumn{2}{|l|}{ Total des étudiants des 3 universités } & $\mathrm{N}$ & Moyenne & Ecart type & Oui & Non \\
\hline \multicolumn{2}{|l|}{ Ea- Une voiture se déplace sur un sol horizontal } & 101 & 1,59 & 0,494 & $40 \%$ & $60 \%$ \\
\hline \multicolumn{2}{|c|}{ Eb- Un point matériel se déplace à vitesse constante } & 101 & 1,51 & 0,502 & $48 \%$ & $52 \%$ \\
\hline \multicolumn{2}{|c|}{ Ec- Un dispositif permettant l'étude de la chute libre } & 101 & 1,29 & 0,455 & $71 \%$ & $29 \%$ \\
\hline \multicolumn{2}{|c|}{ Ed- Diagramme de vitesse d'un mouvement rectiligne } & 101 & 1,63 & 0,484 & $37 \%$ & $63 \%$ \\
\hline \multicolumn{2}{|l|}{ Ee- La relation mathématique $\mathrm{F}=\mathrm{ma}$} & 101 & 1,56 & 0,498 & $44 \%$ & $56 \%$ \\
\hline
\end{tabular}

Résultats de l'item 2.a : L'identification du modèle et la cohérence de sa perception. 


\section{ANNEXE C}

\section{Les résultats de l'indicateur relatif à l'identification du modèle et la cohérence de sa perception}

\section{Les exemples qui ne représentent pas des modèles}

\begin{tabular}{|c|c|c|c|c|c|}
\hline Item 2.b & Université & Moyenne & Ecart type & Oui & Non \\
\hline \multirow[t]{3}{*}{ E'a- Le soleil émet de la lumière. } & Médéa & 1,20 & 0,405 & $80 \%$ & $20 \%$ \\
\hline & Blida & 1,38 & 0,492 & $63 \%$ & $37 \%$ \\
\hline & Kouba & 1,24 & 0,435 & $76 \%$ & $24 \%$ \\
\hline \multirow{3}{*}{$\begin{array}{l}\text { E'b- La réflexion d'un rayon lumineux } \\
\text { arrivant sur un dioptre. }\end{array}$} & Médéa & 1,40 & 0,496 & $60 \%$ & $40 \%$ \\
\hline & Blida & 1,91 & 0,296 & $10 \%$ & $90 \%$ \\
\hline & Kouba & 1,38 & 0,494 & $62 \%$ & $38 \%$ \\
\hline \multirow{3}{*}{$\begin{array}{l}\text { E'c- Ensemble de boules permettant } \\
\text { l'étude des chocs. }\end{array}$} & Médéa & 1,60 & 0,496 & $40 \%$ & $60 \%$ \\
\hline & Blida & 1,78 & 0,420 & $22 \%$ & $78 \%$ \\
\hline & Kouba & 1,69 & 0,471 & $31 \%$ & $69 \%$ \\
\hline \multirow{3}{*}{$\begin{array}{l}\text { E'd- Schéma d'un solide suspendu à un } \\
\text { ressort vertical }\end{array}$} & Médéa & 1,70 & 0,405 & $20 \%$ & $80 \%$ \\
\hline & Blida & 1,81 & 0,420 & $22 \%$ & $78 \%$ \\
\hline & Kouba & 1,76 & 0,435 & $24 \%$ & $76 \%$ \\
\hline \multirow{3}{*}{$\begin{array}{l}\text { E'e- L'équation horaire d'un mouvement } \\
\text { rectiligne : } x=v t+x_{0}\end{array}$} & Médéa & 1,55 & 0,504 & $45 \%$ & $55 \%$ \\
\hline & Blida & 1,56 & 0,504 & $44 \%$ & $56 \%$ \\
\hline & Kouba & 1,66 & 0,484 & $34 \%$ & $66 \%$ \\
\hline Total des étudiants des 3 universités & $\mathrm{N}$ & Moyenne & Ecart type & Oui & Non \\
\hline E'a- Le soleil émet de la lumière. & 101 & 1,27 & 0,445 & $73 \%$ & $27 \%$ \\
\hline $\begin{array}{l}\text { E'b- La réflexion d'un rayon lumineux arrivant } \\
\text { sur un dioptre. }\end{array}$ & 101 & 1,55 & 0,500 & $45 \%$ & $55 \%$ \\
\hline $\begin{array}{l}\text { E'c- Ensemble de boules permettant l'étude des } \\
\text { chocs }\end{array}$ & 101 & 1,68 & 0,468 & $32 \%$ & $68 \%$ \\
\hline $\begin{array}{l}\text { E'd- Schéma d'un solide suspendu à un ressort } \\
\text { vertical }\end{array}$ & 101 & 1,75 & 0,434 & $25 \%$ & $75 \%$ \\
\hline $\begin{array}{l}\text { E'e- L'équation horaire d'un mouvement } \\
\text { rectiligne : } x=v t+x_{0}\end{array}$ & 101 & 1,58 & 0,495 & $42 \%$ & $58 \%$ \\
\hline
\end{tabular}

Résultats de l'item 2.b : L'identification du modèle et la cohérence de sa perception. 